\title{
Band-like motion and mobility saturation in organic molecular semiconductors
}

\author{
S. Fratini ${ }^{1,2}$ and S. Ciuchi ${ }^{3}$ \\ ${ }^{1}$ Institut Néel - CNRS \& Université Joseph Fourier BP 166, F-38042 Grenoble Cedex 9, France \\ ${ }^{2}$ Instituto de Ciencia de Materiales de Madrid, CSIC, \\ Sor Juana Inés de la Cruz 3, E-28049 Madrid, Spain \\ ${ }^{3}$ SMC-INFM Research Center, CNISM and Dipartimento di Fisica \\ Università dell'Aquila, via Vetoio, I-67010 Coppito-L'Aquila, Italy
}

(Dated: November 20, 2018)

\begin{abstract}
We analyze a model that accounts for the inherently large thermal lattice fluctuations associated to the weak van der Waals inter-molecular bonding in crystalline organic semiconductors. In these materials the charge mobility generally exhibits a "metallic-like" power-law behavior, with no sign of thermally activated hopping characteristic of carrier self-localization, despite apparent mean-freepaths comparable or lower than the inter-molecular spacing. Our results show that such puzzling transport regime can be understood from the simultaneous presence of band carriers and incoherent states that are dynamically localized by the thermal lattice disorder.
\end{abstract}

PACS numbers:

In the past years, the development of crystal growing techniques has led to the production of molecular organic semiconductors with extremely low structural disorder, attaining an upper intrinsic limit of the carrier mobility $\mu \sim 10^{1}-10^{2} \mathrm{~cm}^{2} / \mathrm{Vs}$, whose explanation still challenges the scientific community. 1] Surprisingly, despite decades of active investigation there is still no well-established theory of charge transport in these materials. Attempts to generalize the usual concepts that successfully apply to inorganic semiconductors have revealed unsatisfactory, failing to provide a complete understanding of the fundamental mechanisms governing the charge mobility. In this respect, there has been historically a duality between a conventional band-like description [2, 3] relying on the existence of well defined "Bloch" states and the opposite view of self-localized (polaronic) carriers hopping incoherently from molecule to molecule, [4, 5, , 6, , 7] but no conclusive agreement has been reached if either of these limiting descriptions applies in practice. In fact, the most puzzling feature of charge transport in crystalline organic semiconductors is that they generally exhibit a "band-like" mobility characterized by a power-law decrease with temperature, [1, 8] but with absolute values close to or even below the MottIoffe-Regel limit around room temperature, i.e. with apparent mean-free-paths that are comparable or even lower than the inter-molecular spacing. [9] This observation clearly suggests a breakdown of conventional band behavior and has been often taken as an indication of polaronic carrier localization, even though the absence of a distinctive thermally activated mobility is at odds with this interpretation. Recently, an alternative mechanism has been suggested where the electronic wavefunction is localized by the large thermal lattice fluctuations [10, 11] rather than by polaronic self-trapping effects, a picture that is indeed compatible with the observed mobilities, $d \mu / d T<0$. However, new experimental evidence on the existence of band-like carriers has gathered from different techniques such as transient photoconductivity, 12, 13 optical absorption [14, 15] and photoemission [16], which has once more reopened the debate.

In this Letter, we revisit the traditional duality of band-like vs. localized carriers in highly conducting crystalline organic semiconductors, where electronlattice interactions are too weak to induce polaronic selflocalization. In these systems the limiting paradigm of thermally activated hopping is replaced by a different high temperature regime where the carriers diffuse incoherently in the presence of large thermal lattice fluctuations, giving rise to a weakly metallic behavior which is analogous to the "resistivity saturation" characteristic of bad metals. 17, 18] In the experimentally accessible temperature range, our results show that both band-like features characteristic of Bloch electrons and incoherent states of a more localized nature are simultaneously present in the single-particle excitation spectrum, albeit at different energy scales. Accordingly, different experimental probes will see alternatively one feature or the other, which is likely at the origin of the longstanding controversy on the microscopic identity of the charge carriers in these materials. Because of this duality, the charge dynamics cannot be understood in terms of a single microscopic mechanism, since band-like carriers as well as incoherent excitations of a more localized nature both contribute to the electrical mobility.

In organic semiconductors the molecules are bound together by weak van der Waals forces. These are at the origin of the most salient characteristics of these materials, that are held responsible for their peculiar electronic properties: the presence of extremely narrow electronic bandwidths, comparable to the thermal energy at room temperature, and their mechanical softness, reflected in large fluctuations of the molecular lattice around its equilibrium structure. The thermal lattice vibrations give rise to a large (dynamic) disorder in the inter-molecular transfer integrals $t$ which is cur- 
rently identified as the dominant intrinsic limiting factor of the mobility in these compounds. [5, 10, 19, 20, 21] Other microscopic mechanisms, such as the reorganization of electronic states within the molecules (the intramolecular electron-phonon interaction), have been shown to have a weaker effect on charge transport, [10, 20] especially in crystals of large molecules such as pentacene and rubrene where the highest mobilities are currently achieved [25, 26]. The above features can be formalized in the following model Hamiltonian [2, 3, 5, 10, 22]

$$
\begin{aligned}
H & =-t \sum_{i} f\left(X_{i}-X_{i+\delta}\right)\left(c_{i}^{+} c_{i+\delta}+c_{i+\delta}^{+} c_{i}\right) \\
& +\sum_{i} \frac{M \omega_{0}^{2}}{2} X_{i}^{2}
\end{aligned}
$$

where $c_{i}^{+}\left(c_{i}\right)$ are creation (annihilation) operators for an electron on a given molecule and the function $f$ measures the variation of the transfer integrals between neighboring molecules (at sites $i$ and $i+\delta$ ) due to their relative displacements, $X_{i}-X_{i+\delta}$. In the following we shall assume for clarity a linear dependence $f(x)=1-\alpha x$, although our method can be easily generalized to other forms of $f(x)$. 27]

To solve the model Eq. (11) we treat the $X_{i}$ as classical variables, assuming that the lattice dynamics are slower than any other time-scale in the system. This is justified in virtue of the extremely low frequencies of the inter-molecular phonons that couple to the electronic motion, smaller than both the thermal and the band energy scale $\left(\hbar \omega_{0} \simeq 4-9 \mathrm{meV}, t \simeq 130 \mathrm{meV}\right.$ in rubrene 20], see [10, 19, 24] for different compounds). In this case the electronic properties depend on a single dimensionless coupling parameter $\lambda=\alpha^{2} t /\left(2 M \omega_{0}^{2}\right)$. We evaluate the non-local electron Green's function $G(i, j, \omega, X)$ $(i, j=$ lattice sites) for a statistical set of lattice configurations $X=\left\{X_{i}\right\}$ corresponding to a random extraction of the molecular displacements $X_{i}$ out of a gaussian distribution $P\left(X_{i}\right) \propto \exp \left(-M \omega_{0}^{2} X_{i}^{2} / 2 k_{B} T\right)$. The electronic problem at each given $X$ is solved numerically using an algorithm based on regularization of recursion formulas [28] as an alternative to common exact diagonalization techniques, so that system sizes up to $N=2^{16}$ sites can be achieved. In order to control the residual finite-size effects we attach infinite metallic leads to both ends of the linear chain. The dynamical nature of the lattice disorder is restored upon averaging over up to $10^{6}$ lattice configurations $X$, defining the physically observable Green's function as $G(|i-j|, \omega)=\langle G(i, j, \omega, X)\rangle_{X}$. The electrical conductivity is then evaluated via the Kubo formula expressed in terms of the exact electron propagators, neglecting vertex corrections as was done in Ref. [17] and validated in Ref. [18] in a model for metallic fullerenes. This procedure allows to establish a direct connection between the excitation spectrum and the transport properties, capturing the essential aspects of the transport
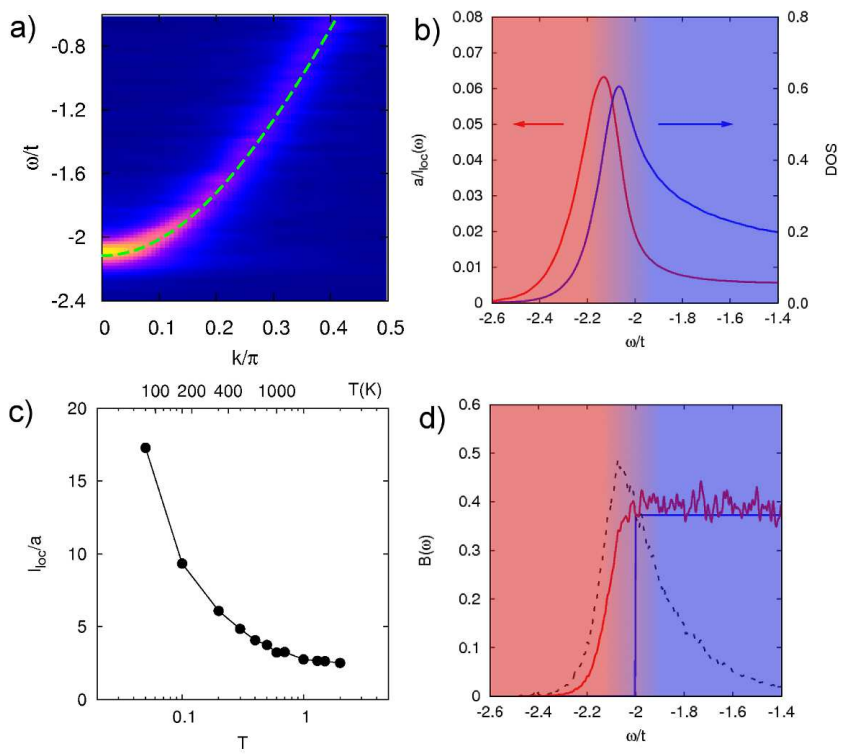

FIG. 1: Quantities calculated from the solution of the model Eq.(1), representative for crystalline rubrene at room temperature $(T=0.2 t$ with $t=143 \mathrm{meV}$ and $\lambda=0.17$ as taken from Ref.[20]). a) spectral function $A(k, \omega)$, showing a well defined band with a weakly renormalized dispersion; b) energy resolved localization length (red) and density of states (blue); c) spatial extension of the electronic wave-function; d) current-current correlation function (red), and the same function multiplied by the thermal occupation factor as it appears in Eq. (2) (black dashed). The blue line is the Boltzmann result for band electrons, $B(\omega)=\sum_{k} \delta\left(\omega-\epsilon_{k}\right) v_{k}^{2} \tau_{k}$, with $\epsilon_{k}$ the band dispersion and $\tau_{k}$ the quasiparticle lifetime. The data are evaluated with $10^{6}$ statistical samples, respectively on $\left.\left.a), b) N=2^{10}, c\right), d\right) 2^{8}$ lattice sites.

mechanism which stem directly from the dual nature of the electronic states (see below). 31] Here we present results obtained on a one-dimensional molecular chain, ideally corresponding to the direction of highest mobility in the organic crystal.

Fig. 17 shows the single-particle spectral function $A(k, \omega)=-\operatorname{Im} \tilde{G}(k, \omega) / \pi[\tilde{G}(k, \omega)$ being the Fourier transform of the Green's function $G(|i-j|, \omega)]$ that carries information on the energy dispersion and lifetime of the extended Bloch waves. Owing to the relatively low values of $\lambda=0.05-0.2$ in organic semiconductors [10, 19, 20, 24], no polaronic self-trapping is expected. Indeed, at such moderate coupling strengths one obtains a weakly renormalized band dispersion (dashed line) and a quasi-particle scattering rate that increases with temperature, $1 / \tau \propto \lambda\left\langle\left(X_{i}-X_{j}\right)^{2}\right\rangle \sim \lambda k_{B} T$, being proportional to the disorder induced by the thermal lattice motion. We see from Fig. 19 that even at room temperature the scattering rate is sufficiently small compared to the bandwidth to allow for the existence of well-defined Bloch states. Moreover, the quasi-particles appear to be sharper near the band edge, i.e. precisely for those states that would be populated in a clean non-degenerate semi- 
conductor. The states of low-momentum are partly protected by the "off-diagonal" nature of the inter-molecular electron-lattice coupling, i.e. the fact that the dominant interaction acts on the transfer integrals rather than on the molecular energy levels. A perturbative calculation yields $1 / \tau_{k} \propto k$ at low momenta, but our numerical data show that the suppression of scattering is not complete and saturates to a finite value $1 / \tau_{k=0} \simeq \pi \lambda k_{B} T$.

While the $k$-space analysis carried out above would point to a conventional scenario based on weakly scattered momentum states, a different conclusion is reached if one looks at physical quantities in real space. The idea is that if one takes a shapshot of the system at a given time the electronic wave-function will be apparently localized by the instantaneous disordered landscape of lattice deformations, even though the carrier actually diffuses on the long time owing to the dynamics of the molecular lattice (diffusion eventually sets in at times longer than the period of inter-molecular vibrations, which is itself much larger than the timescale of electronic motion). [10, 11] To illustrate this point we calculate the average spread of the electronic wave-function for the states at a given energy, $l_{\mathrm{loc}}(\omega)$. Following Refs. [29, 30], this quantity can be expressed in terms of the density of states (DOS) $\rho(\omega)=G(0, \omega)$ as

$$
l_{l o c}^{-1}(\omega)=\int d \omega^{\prime} \rho(\omega) \rho\left(\omega^{\prime}\right) \log \left(\left|\omega-\omega^{\prime}\right| /\left|\omega^{\prime}\right|\right) .
$$

The comparison of the localization length with the actual DOS in Fig 1b shows that two classes of states of very different nature coexist in the excitation spectrum. The states within the band, $(\omega \gtrsim-2 t)$, are effectively delocalized over hundreds of molecular sites and can therefore be treated in the wave representation. However, the dynamical disorder induced in the inter-molecular transfer integrals by the thermal lattice vibrations gives rise to a tail of incoherent excitations emerging in the DOS below the band edge, $(\omega \lesssim-2 t)$. These states have a much shorter spatial extent and are ultimately responsible for the localized character of the particles: the actual localization length is obtained as the thermal average $l_{\text {loc }}^{-1} \propto \int l_{\text {loc }}^{-1}(\omega) e^{-\omega / k_{B} T}$, which is dominated by such incoherent tail states where $l_{l o c}^{-1}(\omega)$ is maximum. As a result, real-space probes of the electronic properties 32. will point to the presence of particles localized on few molecular sites [10], as shown in Fig. 15. On the other hand, momentum-resolved probes such as angle-resolved photoemission [16] should provide a picture in agreement with the wave nature of electrons, as shown in Fig. 1h.

To ascertain how the duality evidenced in the singleparticle excitation spectrum is reflected in the transport properties, one needs to go beyond the semi-classical Boltzmann treatment which is valid for band-like carriers alone. We therefore resort to the Kubo formula

$$
\mu=\frac{\pi \mu_{0}}{Z k_{B} T} \int d \omega B(\omega) e^{-\omega / k_{B} T}
$$

which expresses the mobility in terms of the currentcurrent correlation function in the limit of zero exchanged momentum, $B(\omega)=\lim _{q \rightarrow 0} \int d \omega e^{i \omega t}\langle[J(t), J(0)]\rangle \quad\left(\mu_{0}=\right.$ $e a^{2} / \hbar$ carries the dimensions of mobility, with $a$ the average inter-molecular spacing, and $\left.Z=\int d \omega \rho(\omega) e^{-\omega / k_{B} T}\right)$. Inspection of Eq. (3) shows that the temperature dependent function $B(\omega)$ represents the contribution to the electrical conduction from the states at energy $\omega$, which can be viewed as an "energy-resolved" carrier mobility. The current-current correlation function calculated from the exact electron propagators clearly shows that conduction from the band-like carriers seen in the spectral function of Fig. 1h is reasonably well described by Boltzmann theory, that predicts a constant $B(\omega)$ within the band, see Fig. 11. However, an additional transport channel emerges due to the states below the band edge, corresponding to the incoherent tail seen in the DOS of Fig. 10. Both band-like and incoherent states are therefore expected to contribute to the transport mechanism. Their relative role will depend crucially on the temperature, being essentially controlled by the amount of thermal lattice disorder (which sets the size of the incoherent tail) and to a lesser extent by their respective thermal population, via the exponential term in Eq.(3). This interplay is best visualized by looking directly at the integrand of Eq. (3), i.e. the bubble $B(\omega)$ weighted by the Boltzmann factor (black dashed curve in $1 \mathrm{~d}$ ): at $T=0.2 t$ most of the weight is located right across the energy scale that separates the dynamically localized from the delocalized states. Upon increasing the temperature, the relative weight of the incoherent states progressively increases up to the point where band states get competely washed out, as their lifetime becomes shorter than the inter-molecular transfer time $\tau \lesssim t^{-1}$.

The mobility obtained from Eq. (3) is illustrated in Fig. 2 together with the limiting results valid for band electrons alone $\left(\mu \sim T^{-3 / 2}\right.$ and $\sim T^{-2}$ are obtained from the Boltzmann treatment at $k_{B} T \lesssim 2 t$ and $k_{B} T \gtrsim 2 t$, see Refs. [2, 3]) and for the random diffusion of fully incoherent states [4, 23]. The latter corresponds to the regime of "mobility saturation" 17, 18, 33 that sets in when the the mean-free path for band electrons falls below the inter-molecular spacing, $l_{\mathrm{mfp}} \lesssim a$, leading to a complete loss of momentum conservation. Above this temperature, which essentially coincides with the condition $\tau \lesssim t^{-1}$ given above, the charge transport proceeds via the incoherent diffusion of carriers being randomized at each jump as in a classical random walk. This mechanism results in a rather flat "metallic-like" power-law behavior [4, 23], $\mu \sim T^{-1 / 2}$, that should be contrasted with the exponentially activated behaviour characteristic of self-trapped polarons, occurring for much larger values of the electron-lattice interaction, $\lambda \gtrsim 1$. We see from Fig. 2 that the calculated mobility undergoes a very broad crossover where it progressively interpolates 


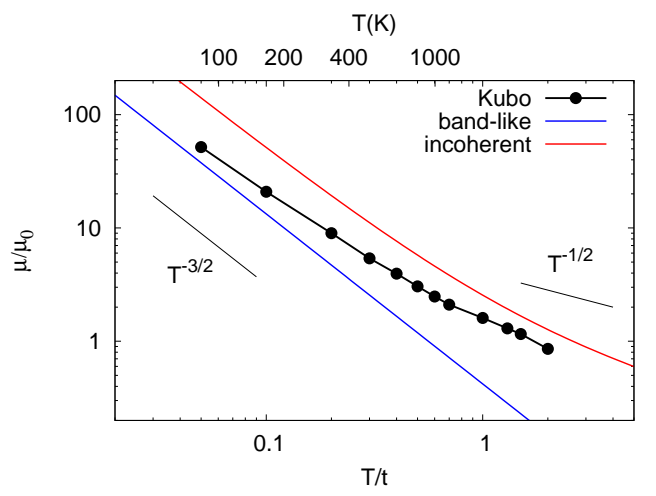

FIG. 2: Mobility calculated from the Kubo formula in terms of the electron propagators obtained from the numerical simulation. For realistic bandwidth and electron-lattice interaction parameters for organic semiconductors (see Fig. 1), both band-like conduction and incoherent diffusion contribute to the transport mechanism in the relevant temperature range around room temperature.

between the band-like regime and the incoherent diffusion regime. In the temperature range of interest, these two competing mechanisms are simultaneously present and combine as independent transport channels. 33, 34]

Our results based on a model that incorporates the essential ingredients relevant for crystalline organic semiconductors show that neither of the two limiting pictures proposed in the past adequately describes the charge dynamics. Firstly, polaronic self-localization is prevented in high-mobility organic semiconductors by the relatively weak electron-lattice interactions. However, the opposite view of band-like transport is not appropriate either: due to the large thermal lattice fluctuations arising from the mechanical softness of these systems, incoherent states having a localized nature emerge and are found to coexist with more conventional Bloch states. Both bandlike and incoherent states actually contribute to the electronic properties of these materials but are expected to show up differently according to the experimental probe. Also, depending on the actual values of the bandwidth and inter-molecular electron-lattice coupling relevant to a given material, a whole range of intermediate behaviours between the two limiting transport scenarios can arise, which might explain the large variability of exponents of the mobility $\mu \sim T^{-n}$ observed in the literature. [1, 8]

[1] M. E. Gershenson, V. Podzorov and A. F. Morpurgo. Rev. Mod. Phys. 78, 973 (2006)

[2] S. H. Glarum, J. Phys. Chem. Solids 24, 1577 (1963)

[3] L. Friedman, Phys. Rev. 140, A1649 (1965)

[4] P. Gosar \& S.-I. Choi, Phys. Rev. 150, 529 (1966)

[5] R. W. Munn \& R. J. Silbey, Chem. Phys. 83, 1854 (1985)
[6] V. M. Kenkre, J. D. Andersen, D. H. Dunlap, and C. B. Duke Phys. Rev. Lett. 62, 1165 (1989).

[7] E. A. Silinsh, A. Klimkans, S. Larsson, V. Capek. Chem. Phys. 198, 311 (1995)

[8] N. Karl, Organic Electronic Materials, ed. R. Farchioni \& G. Grosso, Springer-Verlag, Berlin, pp. 283-326 (2001)

[9] Y. C. Cheng et al., J. Chem. Phys 118, 3764 (2003)

[10] A. Troisi \& G. Orlandi, Phys. Rev. Lett. 96, 086601 (2006)

[11] J. D. Picon, M. N. Bussac, L. Zuppiroli, Phys. Rev. B $75,235106(2007)$

[12] O. Ostroverkhova et al., Appl. Phys. Lett. 88, 162101 (2006)

[13] D. Moses, C. Soci, X. Chi, and A. P. Ramirez. Phys. Rev. Lett. 97, 067401 (2006)

[14] M. Fischer, M. Dressel, B. Gompf A. K. Tripathi and J. Pflaum. Appl. Phys. Lett. 89, 182103 (2006)

[15] Z. Q. Li et al., Phys. Rev. Lett. 99, 016403 (2007)

[16] H. Kakuta et al., Phys. Rev. Lett. 98, 247601 (2007)

[17] A. J. Millis, J. Hu , S. Das Sarma, Phys. Rev. Lett. 82, 2354 (1999)

[18] O. Gunnarsson \& J.E. Han, Nature 405, 1027 (2000)

[19] K. Hannewald \& P. A. Bobbert. Phys. Rev. B 69, 075212 (2004).

[20] A. Troisi, Adv. Mat. 19, 2000 (2007)

[21] H. A. Laarhoven et al., J. Chem. Phys. 129, 044704 (2008)

[22] A. Madhukar \& W. Post, Phys. Rev. Lett. 39, 1424 (1977)

[23] H. J. Sumi, Chem. Phys. (1979)

[24] L. J. Wang, Q. Peng, Q. K. Li, and Z. Shuai. J. Chem. Phys. 127, 044506 (2007)

[25] A. Devos \& M. Lannoo, Phys. Rev. B 58, 8236 (1998)

[26] V. Coropceanu et al., Phys. Rev. Lett. 89, 275503 (2002)

[27] The validity of the linear approximation can be justified by observing that in the materials under study the statistical distribution of transfer integrals essentially follows the gaussian shape determined by the thermal distribution of lattice displacements (see e.g. Fig.1a of Ref. [20]), while more general non-linear couplings would lead to markedly asymmetric and non-gaussian distributions.

[28] H. A. Yamani \& M. S. Abdelmonem, J. Phys. A: Math. Gen., 30, 2889 (1997)

[29] D. J. Thouless, J. Phys. C 5, 77 (1972)

[30] G. Theodorou \& M. H. Cohen, Phys. Rev. B 13, 4597 (1976)

[31] Dropping vertex corrections is physically meaningful as it discards precisely those quantum interference effects that would lead to genuine electron localization in the presence of static disorder, but are absent for the dynamical lattice disorder considered here, see e.g. [P. A. Lee \& T. V. Ramakrishnan, Rev. Mod. Phys. 57, 287 (1985)]

[32] K. Marumoto, S. I. Kuroda, T. Takenobu, Y. Iwasa, Phys. Rev. Lett. 97, 256603 (2006)

[33] M. Calandra \& O. Gunnarsson, Phys. Rev. B 66, 205105 (2002)

[34] S. Fratini \& S. Ciuchi, Phys. Rev. Lett. 91, 256403 (2003)

S. F. acknowledges financial support from MICINN (Consolider CSD2007-00010) and from the the Comunidad de Madrid (CITECNOMIK). S. C. aknowledges the hospitality of ICMM-CSIC. 